\title{
Modélisation du comportement effectif du combustible MOX par une analyse micromécanique en champs de transformation non uniformes
}

\author{
Rodrigue Largenton ${ }^{1, a}$, Jean-Claude Michel ${ }^{2}$, Pierre Suquet $^{2}$ et Renaud Masson ${ }^{3}$ \\ 1 Dpt MMC EDF R\&D, Site des Renardières, Avenue des Renardières, 77818 Moret-sur-Loing, France \\ 2 LMA CNRS, 31 chemin Joseph Aiguier, 13402 Marseille Cedex 20, France \\ 3 CEA, DEN, DEC, Cadarache, 13108 Saint-Paul-Lez-Durance Cedex, France
}

Reçu le 22 avril 2011, accepté le 22 juin 2011

\begin{abstract}
Résumé - La répartition hétérogène du plutonium dans le combustible nucléaire MOX implique que la modélisation de son comportement, durant l'irradiation en réacteur à eau pressurisée, passe par la construction de lois macroscopiques faisant intervenir les effets microstructuraux. Pour homogénéiser ce problème local, nous avons choisi une méthode d'analyse par champs de transformation non uniformes, abrégé anglais NTFA (J.-C. Michel, P. Suquet, Int. J. Solids Struct. 40 (2003) 6937-6955). Nous présentons donc ici, les travaux menés sur ce modèle pour l'adapter au comportement du MOX en service, ainsi qu'une comparaison des résultats globaux et locaux de ce modèle à des calculs de référence.
\end{abstract}

Mots clés : Combustible / nucléaire / MOX / microstructure / hétérogène / homogénéisation / NTFA

\begin{abstract}
The MOX fuel effective behavior in nominal pressure water reactor condition: Micro-mechanical modeling by non uniform transformation field analysis The heterogeneous distribution of the plutonium in the MOX nuclear fuel involves that the modeling of its behavior, under irradiation in pressure water reactor (PWR), is realized by the construction of macroscopic laws taking into account the microstructural effects. To homogenize this local problem, we chose a Non uniform Transformation Field Analysis (J.-C. Michel, P. Suquet, Int. J. Solids Struct. 40 (2003) 6937-6955). We thus present here, the works led on this model to adapt it to the MOX fuel behavior in PWR nominal conditions, as well as a comparison of the global and local results of this model to reference calculations.
\end{abstract}

Key words: MOX / nuclear fuel / heterogeneousness / homogenisation / NTFA

\section{Introduction}

La répartition hétérogène du plutonium dans le combustible nucléaire MOX implique que ce matériau est un composite constitué de deux ou trois phases. Pour les combustibles MOX actuels, on observe typiquement trois phases :

- une phase inclusionnaire à forte concentration en plutonium, nommée les amas plutonifères;

- une autre phase inclusionnaire à faible teneur en plutonium, nommée les amas uranifères;

- et une phase d'enrobage à teneur modérée en plutonium.

Par conséquent, la compréhension du comportement du combustible MOX, durant l'irradiation, passe par

\footnotetext{
a Auteur pour correspondance :

rodrigue. largenton@edf.fr
}

la construction de lois macroscopiques faisant intervenir les effets microstructuraux. Pour homogénéiser ce problème local, nous avons choisi une méthode d'analyse par champs de transformation non uniformes [1]. Les modèles NTFA se nourrissant de modes issus de calculs de microstructure, nous avons, tout d'abord en amont, développé des outils permettant de représenter géométriquement et numériquement le combustible MOX en 3D à partir d'images expérimentales 2D. Ces travaux sont détaillés dans [2] et une représentation numérique 3D du MOX, réalisée à l'aide de ces outils, est présentée dans la première partie de ce document. Dans la deuxième partie, nous présentons les développements théoriques (extension au 3D, prise en compte de déformation libre) et numériques (identification des modes plastiques les plus pertinents) menés sur le modèle NTFA pour l'adapter au comportement du MOX en service. Enfin, une comparaison des résultats macroscopiques et locaux de ce modèle 

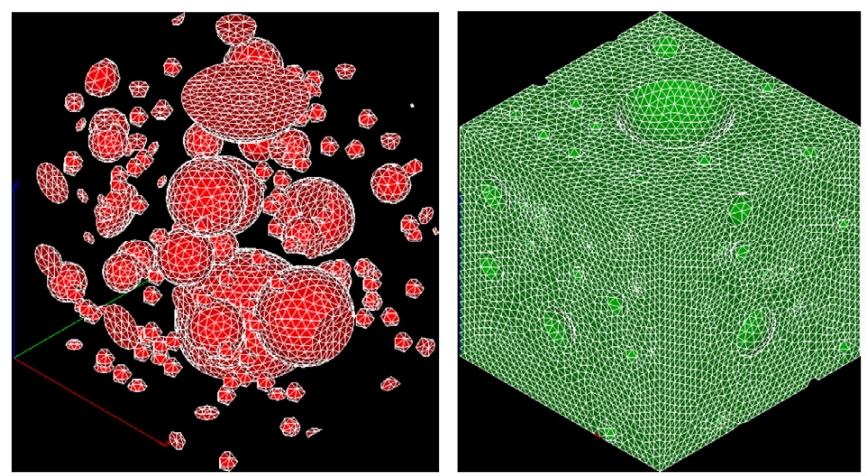

Fig. 1. Maillage et géométrie périodique du combustible MOX en 3D - 99257 tétraèdres. (a) Amas plutonifères 121 particules. (b) Matrice : enrobage plus amas uranifères.

à des calculs de référence par éléments-finis (FEM) est exposée dans la troisième partie.

\section{Représentation numérique 3D du combustible MOX}

Dans cette partie, nous présentons une représentation numérique 3D possible du MOX (cf. Fig. 1), obtenue à l'aide des outils développés dans [2].

Quelques remarques:

- la représentation géométrique ainsi que le maillage sont périodiques ;

- il est important de souligner qu'ici nous ne modélisons que la phase inclusionnaire plutonifère. Rien n'empêche de modéliser la phase uranifère avec les outils développés dans [2], mais dans le cadre de cette étude, nous avons pris le parti d'intégrer la phase uranifère dans l'enrobage. Cette phase sera désormais nommée : matrice;

- enfin, la fraction volumique des inclusions plutonifères est de $15 \%$ et le diamètre des inclusions est compris entre $10 \mu \mathrm{m}$ et $70 \mu \mathrm{m}$. Dans la suite de ce document, l'ensemble des résultats numériques présentés portera donc sur cette microstructure.

\section{NTFA : développements théoriques et numériques pour simuler le comportement du combustible MOX en service}

\subsection{Problème local}

En service, la déformation locale en un point $\underline{x} \mathrm{du}$ VER MOX, qui dépend du temps $t$, peut être décomposée de la façon suivante :

$$
\underline{\underline{\varepsilon}}(\underline{x}, t)=\underline{\underline{\varepsilon}}^{\mathrm{e}}(\underline{x}, t)+\underline{\underline{\varepsilon}}^{\mathrm{vp}}(\underline{x}, t)+\underline{\underline{\varepsilon}}^{\mathrm{gs}}(\underline{x}, t)
$$

avec $\underline{\underline{\varepsilon}}^{\mathrm{e}}$ la déformation élastique, $\underline{\underline{\varepsilon}}^{\mathrm{vp}}$ la déformation visqueuse déviatorique traduisant le fluage induit par l'irradiation et $\underline{\underline{\varepsilon}}^{g s}$ la déformation libre traduisant le mécanisme de gonflement solide. Celle-ci est considérée comme une déformation hydrostatique isotrope, uniforme par phase et linéaire en fonction du temps. Le problème mécanique local à résoudre, à un instant $t$, est le suivant :

$$
\left\{\begin{aligned}
\underline{\operatorname{div}} \underline{\underline{\sigma}}(\underline{x}, t) & =0, \underline{\underline{\sigma}}(\underline{x}, t)=\underline{\underline{\underline{L}}}(\underline{x}): \\
& \times\left(\underline{\underline{\varepsilon}}(\underline{x}, t)-\underline{\underline{\varepsilon}}^{\mathrm{gs}}(\underline{x}, t)-\underline{\underline{\varepsilon}}^{\mathrm{vp}}(\underline{x}, t)\right) \\
\underline{\underline{\varepsilon}}^{\mathrm{gs}}(\underline{x}, t)= & \sum_{r=1}^{N} \chi^{(r)}(\underline{x}) \varepsilon_{r}^{\mathrm{gs}}(t) \underline{\underline{i}}, \underline{\underline{\varepsilon}}^{\bullet \mathrm{vp}}(\underline{x}, t) \\
= & \sum_{r=1}^{N} \chi^{(r)}(\underline{x}) \frac{\partial \psi_{r}}{\partial \underline{\underline{\sigma}}}\left(\sigma_{\mathrm{eq}}(\underline{x}, t)\right) \\
\psi_{r}\left(\sigma_{\mathrm{eq}}\right)= & \frac{1}{2} A_{\text {irr }} F_{r} \cdot \sigma_{\text {eq }}^{2} e^{-\frac{Q_{i r r}}{R T}} \\
\underline{u}(\underline{x}, t)= & \underline{\underline{E}}(t) \cdot \underline{x}+\underline{\tilde{u}}(\underline{x}, t), \underline{\tilde{u}}(\underline{x}, t) \\
& \text { périodique et } \underline{\underline{\sigma}} \underline{x}, t) \cdot \underline{n}(\underline{x}) \text { antipériodique }
\end{aligned}\right.
$$

avec :

- $\chi^{(r)}(\underline{x})$ la fonction caractéristique associée à la phase $r, \underline{\underline{i}}$ le tenseur identité d'ordre 2 et $\varepsilon_{r}^{\mathrm{gs}}(t)$ la déformation libre scalaire de gonflement solide uniforme associée à la phase $r$ et $N$ le nombre de phases;

- $F_{r} \bullet$ la densité de fission moyenne dans la phase $r, T$ la température et $R, Q_{\mathrm{irr}}, A_{\mathrm{irr}}$ des constantes.

La vitesse de gonflement solide $\varepsilon_{\mathbf{g s}^{r}}^{\bullet}$ et la densité de fission $F_{r} \bullet$ sont différentes d'une phase à l'autre car la teneur en plutonium fissile y est différente. En service, le comportement du combustible MOX est donc élasto-viscoplastique linéaire incompressible avec déformation libre (l'incompressibilité ne touche que la partie viscoplastique).

\subsection{Analyse par champs de transformation non uniformes : principe}

\subsubsection{Champs de transformation non uniformes}

Pour homogénéiser ce problème local, nous avons choisi une méthode d'analyse par champs de transformation non uniformes [1]. Avec cette approche, la déformation locale viscoplastique est décomposée sous la forme :

$$
\underline{\underline{\varepsilon}}^{\mathrm{vp}}(\underline{x}, t)=\sum_{k=1}^{M} \underline{\underline{\mu}}^{\mathrm{k}}(\underline{x}) \varepsilon_{k}^{\mathrm{vp}}(t)
$$

avec :

- $\underline{\underline{\mu}}^{k}(\underline{x})$ un ensemble de champs tensoriels non uniformes, appelés modes plastiques. Le choix des modes plastiques est réalisé sous différents chargements élémentaires (cf. Sect. 3.3); 
$-\varepsilon_{k}^{\mathrm{vp}}(t)$ la déformation viscoplastique associée au mode $k$.

Avec cette approximation (3), on passe d'un nombre infini de variables internes qui étaient tensorielles à un nombre fini $M$ de variables scalaires. Il est important de remarquer que le nombre $M$ de modes plastiques peut être différent du nombre de constituants $N$. D'autres hypothèses sont faites pour simplifier la théorie [1] :

1. Le support de chaque mode est entièrement contenu dans une seule phase du matériau.

2. Pour un comportement incompressible, les modes sont des champs tensoriels à trace nulle.

3. Les modes sont orthogonaux : $\left\langle\underline{\mu}^{k}: \underline{\mu}^{l}\right\rangle=0$ si $k \neq l$. Cette condition est évidemment vérifiée quand les modes ont leur support dans des phases différentes, mais doit être imposée pour les modes qui ont leur support dans la même phase.

4. Enfin, les modes sont normalisés : $\left\langle\mu_{\mathrm{eq}}^{k}\right\rangle=1$ où $\mu_{\mathrm{eq}}^{k}=$ $\left(\frac{2}{3} \underline{\underline{\mu}}^{k}: \underline{\underline{\mu}}^{k}\right)^{1 / 2}$.

Avec l'approximation (3), le champ de déformation locale est donné à l'instant $t$ par :

$$
\begin{aligned}
\underline{\underline{\varepsilon}}(\underline{x}, t)=\underline{\underline{\underline{A}}}(\underline{x}): \underline{\underline{E}}(t)+\sum_{l=1}^{M} & \left(\underline{\underline{\underline{D}}} * \underline{\underline{\mu}}^{l}\right)(\underline{x}) \varepsilon_{l}^{\mathrm{vp}}(t) \\
& +\sum_{r=1}^{N}\left(\underline{\underline{\underline{D}}} * \chi^{(r)} \underline{\underline{i}}\right)(\underline{x}) \varepsilon_{r}^{\mathrm{gs}}(t)
\end{aligned}
$$

où $\underline{\underline{\underline{A}}}(\underline{x})$ est le tenseur élastique de localisation de la déformation élastique, * est le produit de convolution et $\underline{\underline{\underline{D}}}\left(\underline{x}, \underline{x}^{\prime}\right)$ l'opérateur non local qui donne, à déformation moyenne nulle, la déformation au point $\underline{x}$ créée par une prédéformation au point $\underline{x}^{\prime} \cdot \underline{\underline{\underline{\underline{D}}}}\left(\underline{x}, \underline{x}^{\prime}\right)$ est défini par $\underline{\underline{\underline{D}}}\left(\underline{x}, \underline{x}^{\prime}\right)=\underline{\underline{\underline{\Gamma}}}\left(\underline{x}, \underline{x}^{\prime}\right): \underline{\underline{\underline{L}}}\left(\underline{x^{\prime}}\right)$, avec $\underline{\underline{\underline{\underline{\Gamma}}}}\left(\underline{x}, \underline{x^{\prime}}\right)$ l'opérateur de Green élastique non local du milieu hétérogène.

\subsubsection{Variables réduites}

En double contractant (4) par $\underline{\underline{\mu}}^{k}$ et en moyennant sur le VER, on obtient :

$$
e_{k}=\underline{\underline{a_{k}}}+\sum_{l=1}^{M} D_{k l} \varepsilon_{l}^{\mathrm{vp}}(t)+\sum_{r=1}^{N} H_{k r} \varepsilon_{r}^{\mathrm{gs}}(t)
$$

où la déformation réduite $e_{k}$ est égale à $\left\langle\underline{\underline{\varepsilon}}: \underline{\underline{\mu}}^{k}\right\rangle$, le tenseur de localisation réduit $\underline{\underline{a_{k}}}$ vaut $\left\langle\underline{\underline{\mu}}{ }^{k}: \underline{\underline{\underline{A}}}\right\rangle$ et les facteurs d'influence $D_{k l}$ sont égaux à $\left\langle\underline{\underline{\mu}}^{k}:\left(\underline{\underline{\underline{D}}} * \underline{\underline{\mu}}^{l}\right)\right\rangle$ et $H_{k r}$ $\grave{a}\left\langle\underline{\underline{\mu}}^{k}:\left(\underline{\underline{\underline{D}}} * \chi^{(r)} \underline{\underline{i}}\right)\right\rangle$.
Par analogie avec l'équation définissant la déformation réduite $e_{k}$, on peut définir la déformation viscoplastique réduite $e_{k}^{\mathrm{vp}}=\left\langle\underline{\underline{\varepsilon}}^{\mathrm{vp}}: \underline{\underline{\mu}}^{k}\right\rangle$ et la contrainte réduite $\tau_{k}=$ $\left\langle\underline{\underline{\sigma}}: \underline{\underline{\mu}}^{k}\right\rangle$.

On remarque que l'ensemble $\left(\varepsilon_{k}^{\mathrm{vp}}\right)_{k=1}, \ldots, M$ peut être remplacé par l'ensemble $\left(e_{k}^{\mathrm{vp}}\right)_{k=1}, \ldots, M$ puisque :

$$
e_{k}^{\mathrm{vp}}=\left\langle\underline{\underline{\mu}}^{k}: \underline{\underline{\mu}}^{k}\right\rangle \varepsilon_{k}^{\mathrm{vp}}
$$

\subsubsection{Relations constitutives pour les variables réduites}

Puisque les tenseurs élastiques des phases sont supposés isotropes et les modes incompressibles, la contrainte réduite $\tau_{k}$ supportée par la phase $r$ est donnée par :

$$
\tau_{k}=2 G^{r}\left(e_{k}-e_{k}^{\mathrm{vp}}\right)
$$

avec $G^{r}$ le module de cisaillement de la phase $r$, support du mode $k$.

Pour résoudre le problème il nous reste à évaluer $e_{k}^{\mathrm{vp}}$. L'équation d'évolution des $e_{k}^{\mathrm{vp}}$ est obtenue en double contractant $\underline{\underline{\varepsilon}}^{\bullet v}(\underline{x})$ par $\underline{\underline{\mu}}^{k}$ et en moyennant sur le VER :

$$
\begin{aligned}
e_{k^{\mathrm{vp}}}=\left\langle\underline{\underline{\varepsilon^{\bullet v p}}}: \underline{\underline{\mu}}^{k}\right\rangle=\frac{3}{2}\left\langle\frac{\partial \psi}{\partial \sigma_{\mathrm{eq}}}\right. & \left.\left(\sigma_{\mathrm{eq}}\right) \frac{{\underline{\underline{\sigma^{\mathrm{dev}}}}}^{\underline{\underline{\mu}}} \underline{\underline{\mu}}^{k}}{\sigma_{\mathrm{eq}}}\right\rangle \\
& =\frac{3}{2} A_{\mathrm{irr}} F_{r} \bullet e^{-\frac{Q_{\mathrm{irr}}}{R T}} \tau_{K}
\end{aligned}
$$

Le système d'équations (5)-(8) compose les relations constitutives effectives du MOX : système d'équations différentielles couplées d'ordre 1. Ce système est résolu le long d'un trajet imposé, soit dans l'espace des contraintes macroscopiques soit dans l'espace des déformations macroscopiques, ce qui donne les variables internes $\varepsilon_{k}^{\mathrm{vp}}$.

La contrainte macroscopique est elle obtenue en moyennant le champ de contrainte local donné par la loi de comportement du problème (2) et en utilisant les expressions (3) et (4), ce qui donne :

$\underline{\underline{\underline{\Sigma}}}=\underline{\underline{\underline{\underline{L}}}}: \underline{\underline{\underline{E}}}+\sum_{k=1}^{M}\left(\left\langle\underline{\underline{\underline{\underline{L}}}}: \underline{\underline{\underline{\underline{D}}}} * \underline{\underline{\underline{\mu}}}{ }^{k}\right\rangle-\left\langle\underline{\underline{\underline{\underline{L}}}}: \underline{\underline{\mu}}^{k}\right\rangle\right) \varepsilon_{k}^{\mathrm{vp}}$

$+\sum_{r=1}^{N}\left(\left\langle\underline{\underline{\underline{\underline{L}}}}: \underline{\underline{\underline{\underline{D}}}} * \chi^{(r)} \underline{\underline{\underline{i}}}\right\rangle-\left\langle\underline{\underline{\underline{\underline{L}}}}: \chi^{(r)} \underline{\underline{\underline{i}}}\right\rangle\right) \varepsilon_{r}^{\mathrm{gs}}, \underline{\underline{\underline{\underline{\tilde{L}}}}}=\langle\underline{\underline{\underline{L}}}: \underline{\underline{\underline{\underline{A}}}}\rangle$

À ce stade, nous avons supposé que les modes plastiques $\left(\underline{\underline{\mu}}^{k}\right)_{k=1, \ldots, M}$ ainsi que les tenseurs d'influence $\left(\underline{\underline{\underline{D}}} * \underline{\underline{\mu}}^{k}\right)_{k=1, \ldots, M}$ et $\left(\underline{\underline{\underline{D}}} * \chi^{(r)} \underline{\underline{i}}\right)_{r=1, \ldots, N}$ étaient identifiés. Nous exposons dans le paragraphe suivant la démarche employée pour les identifier. 


\subsection{Choix des modes plastiques}

Le choix des modes plastiques est un point-clé dans la précision de la méthode. Ils sont déterminés numériquement en simulant la réponse du composite le long de trajets de chargement radiaux dans l'espace des contraintes macroscopiques $\underline{\underline{\Sigma}}(t)=\lambda(t) \underline{\underline{\Sigma}}_{0}$.

Les modes plastiques $\underline{\underline{\theta}}^{k}$ sont calculés jusqu'à $\underline{\underline{E}}$ : $\underline{\underline{\Sigma}}_{0}^{k}=10 \%$. Dans le cadre de cette étude, $\underline{\underline{E^{\bullet}}}: \underline{\underline{\Sigma}}_{0}^{k}$ est fixée à $10^{-2} \mathrm{~s}^{-1}$. En $3 \mathrm{D}$ et pour le comportement modélisé ici, 7 directions de contraintes $\underline{\underline{\Sigma}}_{0}^{k}$ différentes ont été choisies : 3 tractions uniaxiales sans gonflement ( $k=1$ direction 11 , $k=4$ direction 22 , et $k=6$ direction 33), 3 cisaillements purs sans gonflement $(k=2$ direction $12, k=3$ direction 13 et $k=5$ direction 23 ), et un essai sans contrainte macroscopique mais avec gonflement $(k=7)$.

$$
\left\{\begin{array}{l}
{\underline{\underline{\Sigma^{k}}}}_{0}=\underline{e}_{i} \otimes_{s} \underline{e}_{j} \text { avec } k=1, \ldots, 6, i=1, \ldots, 3 \\
\quad \text { et } j=1, \ldots, 3 \\
\underline{\underline{\Sigma}}_{0}^{7}=\underline{\underline{0}} \text { et } \varepsilon_{r}^{\mathrm{gs}} \text { appliquée dans chaque phase }
\end{array}\right.
$$

Les paramètres matériaux qui ont été choisis pour les 6 premiers états de contraintes sont les suivants : pour la phase 1 matricielle nous prenons $\frac{3}{2} A_{\text {irr }} F_{1} \bullet \mathrm{e}^{-\frac{Q_{\text {irr }}}{R T}}=$ $5,18427 \times 10^{-12} \mathrm{~Pa}^{-1} . \mathrm{s}^{-1}$ et $1,55528 \times 10^{-11} \mathrm{~Pa}^{-1} \cdot \mathrm{s}^{-1}$ pour la phase 2 inclusionnaire. Tandis que les propriétés élastiques sont considérées comme homogènes et isotropes : $E=200 \mathrm{GPa}$ et $\nu=0,3$. Notons que le contraste de viscosité entre les amas plutonifères et la matrice est de 3. Pour le $7^{\mathrm{e}}$ état de contrainte, les paramètres élastiques et visqueux précédents ont été reconduits mais, en plus, nous avons rajouté le contraste de vitesse de gonflement solide entre les phases :

$$
\begin{aligned}
& \text { phase1 : } \varepsilon_{1}^{\mathrm{gs} \bullet}=7,0 \times 10^{-05} \mathrm{~s}^{-1}, \\
& \text { phase } 2: \varepsilon_{2}^{\mathrm{gs} \bullet}=2,1 \times 10^{-02} \mathrm{~s}^{-1}
\end{aligned}
$$

Le contraste de gonflement entre les amas plutonifères et la matrice est, ici, de 300. Ce contraste a été volontairement amplifié par rapport à celui auquel le combustible MOX peut être soumis en réacteur, l'objectif étant de démontrer la faisabilité de cette approche quel que soit le contraste entre les phases.

Pour chaque trajet de chargement $\underline{\underline{\Sigma}}_{0}^{k}$, on considère les modes plastiques $\underline{\underline{\theta}}^{k}$ comme des champs de déformations viscoplastiques pour différents niveaux de chargement $\underline{\underline{E}}: \underline{\underline{\underline{\Sigma}}}_{0}^{k}$. Ces modes sont normalisés via $\left\langle\theta_{\mathrm{eq}}^{k}\right\rangle$ au sens de von Mises. Dans notre cas, les modes ont été choisis toutes les $0,4 \mathrm{~s}$. Au final, nous avons donc $M_{T}(r)$ modes $(25$ dans le cadre de cette étude) par trajet de chargement et par phase $r$. On impose (cf. Sect. 3.2.1) aux modes plastiques candidats pour la méthode NTFA d'être orthogonaux deux à deux par la transformation de KarhunenLoève, développée dans [3], encore appelée décomposition orthogonale aux valeurs propres (proper orthogonal decomposition : POD), en réalisant un changement de base pour obtenir de nouveaux modes où l'information contenue est distribuée de façon optimale.

La transformée de Karhunen-Loève consiste à construire une matrice de corrélation $g_{i j}=\left\langle\underline{\theta}^{i}(\underline{x})\right.$ : $\left.\underline{\underline{\theta}}^{j}(\underline{x})\right\rangle_{i, j=1, \ldots, M_{T}(r)}$ à partir des données numériques et à en extraire les vecteurs propres $\underline{v}^{k}$ et les valeurs propres $\lambda^{k}$ afin d'obtenir une nouvelle base de vecteurs appelés modes. À partir de ces vecteurs propres, on construit les vecteurs de la nouvelle base, qu'on appelle dans la NTFA les modes plastiques $\underline{\underline{\mu}}^{k}(\underline{x})_{\left(k=1, \ldots, M_{T}(r)\right)}$ :

$$
\underline{\underline{\mu}}^{k}(\underline{x})=\sum_{l=1}^{M_{T}(r)} v_{l}^{k} \underline{\underline{\theta}}^{l}(\underline{x})
$$

Un autre intérêt de la transformée de Karhunen-Loève réside dans le fait que la magnitude d'une valeur propre correspond à la quantité d'information contenue dans le vecteur propre associé. En supposant que les valeurs propres sont ordonnées de manière décroissante et en prenant en compte la propriété précédente, seuls les $M(r)$ premiers modes correspondant aux plus grandes valeurs propres et vérifiant le critère :

$$
\sum_{k=1}^{M(r)} \lambda_{k} / \sum_{k=1}^{M_{T}(r)} \lambda_{k} \geq \alpha
$$

peuvent être considérés $(\alpha=0,9999)$. La méthode permet donc de rendre les modes orthogonaux et de sélectionner les modes prépondérants ce qui est en adéquation avec le développement d'un « modèle réduit » (un nombre faible de variables internes). Pour illustrer ces propos, la figure 2 montre les modes $\mu_{\text {eq }}^{1}(\underline{x})$, identifiés par phase et pour le premier trajet de chargement élémentaire $\underline{\underline{\Sigma}}_{0}^{1}$. Pour ce trajet de chargement, nous constatons que deux modes par phase ont été sélectionnés via la méthode de KarhunenLoève.

Au final, pour l'ensemble des trajets de chargements élémentaires, le modèle NTFA est composé de 30 modes (15 dans la matrice et 15 dans les inclusions), soit donc 30 variables internes scalaires $\varepsilon_{k}^{\mathrm{vp}}$. Dans la troisième partie de ce document (cf. Sect. 4), les prédictions macroscopiques et locales de ce modèle sont comparées à des calculs de référence (FEM : éléments-finis).

\section{NTFA : validation du modèle}

\subsection{Résultats macroscopiques NTFA vs. FEM}

\subsubsection{Essais d'écrouissage : direction de contrainte $\underline{\underline{\Sigma}}_{0}^{k}$ imposée et $\underline{\underline{E^{\bullet}}}: \underline{\underline{\Sigma}}_{0}^{k}=10^{-2} \mathrm{~s}^{-1}$}

La figure $3 a$ montre les prédictions macroscopiques du modèle NTFA par rapport aux éléments-finis FEM, 


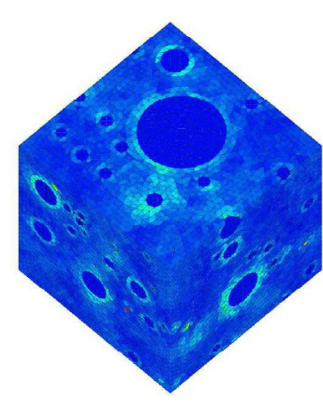

(a)

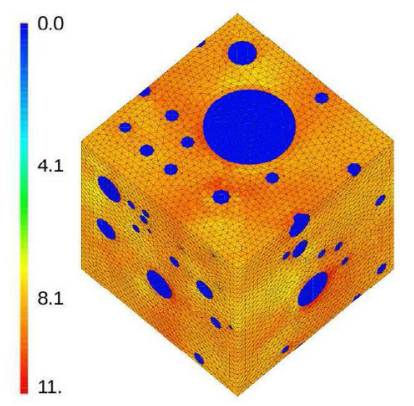

(b)
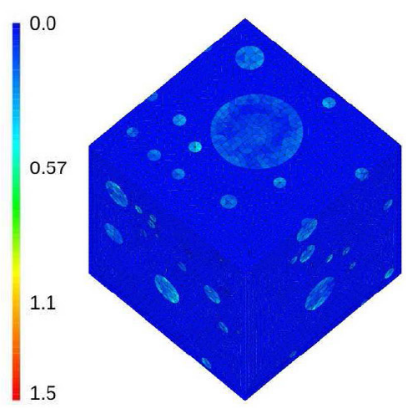

(c)
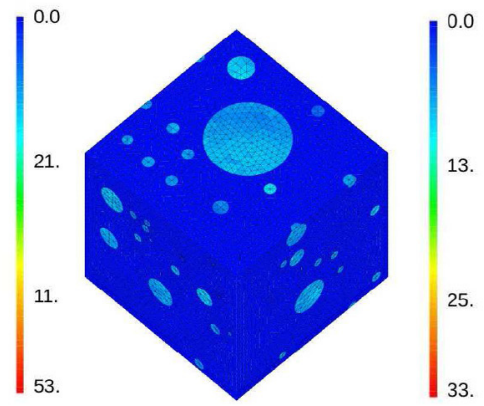

(d)

Fig. 2. Norme équivalente du mode plastique $1: \mu_{\mathrm{eq}}^{1}(\underline{x})$ - trajet de chargement $\underline{\underline{\Sigma}}_{0}^{1}$. (a) mode 1 matrice, (b) mode 2 matrice, (c) mode 1 inclusion, (d) mode 2 inclusion.

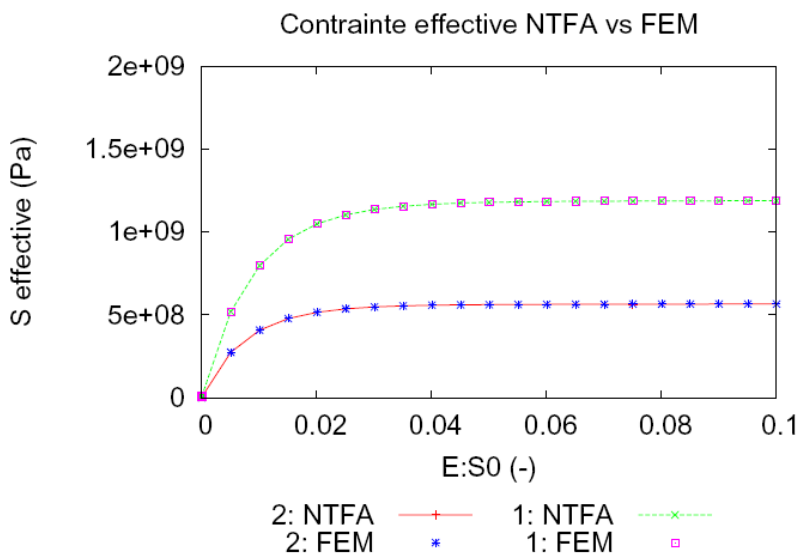

(a)

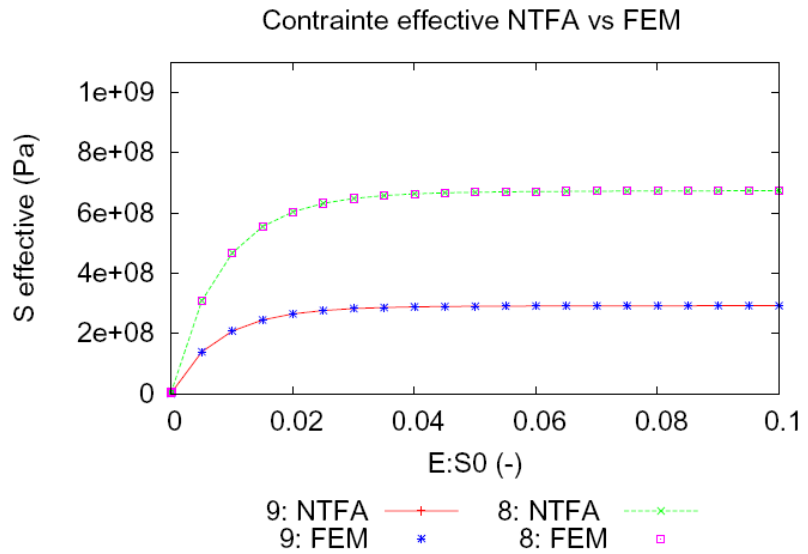

(b)

Fig. 3. Essais d'écrouissage sur la microstructure de la figure 2. (a) Chargements ayant servi pour l'identification des modes; - 1:S0 $=\underline{\underline{\Sigma}}_{0}^{1}-2: S 0=\underline{\underline{\underline{\Sigma}}}_{0}^{2}$. (b) Chargements hors identification des modes; $-8: S 0=\underline{\underline{\underline{\Sigma}}}_{0}^{1}+1 / 2 \underline{\underline{\Sigma}}_{0}^{2} ;-9: S 0=\underline{\underline{\Sigma}}_{0}^{1}+\underline{\underline{\Sigma}}_{0}^{2}$.

pour deux trajets de chargements élémentaires qui ont servi à identifier les modes, à savoir $\underline{\underline{\Sigma}}_{0}^{1}$, nommé 1 , et $\underline{\underline{\Sigma}}_{0}^{2}$, nommé 2. Pour ces deux trajets, nous constatons une très bonne prédiction du modèle NTFA par rapport aux éléments-finis. Cette conclusion n'est pas surprenante puisque il s'agit de trajets ayant servi à identifier les modes.

Sur la figure 3b, nous présentons également les prédictions du modèle NTFA par rapport aux élémentsfinis FEM, pour deux essais, nommés 8 et 9 , qui n'ont pas servi à identifier les modes. Les essais 8 et 9 sont des essais dont la direction de contrainte est une combinaison linéaire de $\underline{\underline{\Sigma}}_{0}^{1}$ et $\underline{\underline{\Sigma}}_{0}^{2}: \underline{\underline{\Sigma}}_{0}^{8}=\underline{\underline{\Sigma}}_{0}^{1}+1 / 2 \underline{\underline{\Sigma}}_{0}^{2}$ et $\underline{\underline{\Sigma}}_{0}^{9}=\underline{\underline{\Sigma}}_{0}^{1}+\underline{\underline{\Sigma}}_{0}^{2}$. Pour ces deux essais, hors identification des modes, nous constatons une très bonne prédiction du modèle NTFA par rapport aux éléments-finis.

\subsubsection{Essais de fluage et de relaxation}

Nous pouvons, également, nous interroger sur la prédiction macroscopique du modèle NTFA pour des essais de type fluage ou relaxation. La figure 4a présente la prédiction macroscopique du modèle NTFA pour un essai de fluage à contrainte uniaxiale (direction 33 $\Sigma_{33}=100 \mathrm{MPa}$ ), tandis que la figure $4 \mathrm{~b}$ présente la prédiction macroscopique du modèle pour un essai de relaxation uniaxiale (direction $33-E_{33}=2 \%$ ). Bien que les modes aient été identifiés pour des essais d'écrouissage, nous constatons toujours une très bonne prédiction du modèle NTFA.

Un intérêt majeur de la méthode NTFA, par rapport à la plupart des approches d'homogénéisation, est qu'elle permet de relocaliser les champs. Nous avons donc également analysé la prédiction locale du modèle NTFA par rapport aux éléments-finis dans la section 4.2.

\subsection{Résultats locaux NTFA vs. FEM}

Les figures 5 a et b illustrent la prédiction du champ de contrainte équivalente de von Mises dans la phase matricielle à $t=10 \mathrm{~s}\left(\underline{\underline{E}}: \underline{\underline{\Sigma}}_{0}^{k}=10 \%\right)$ pour l'essai d'écrouissage numéro 8 de la figure $3 \mathrm{~b}$. 


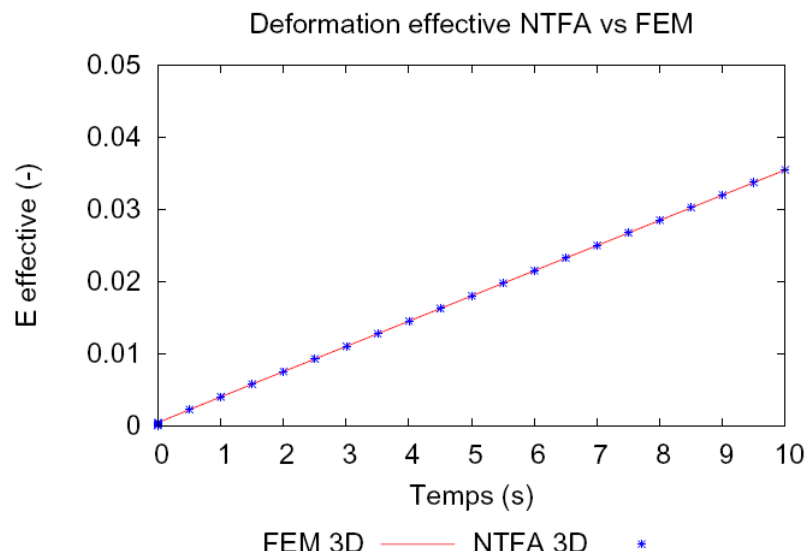

(a)

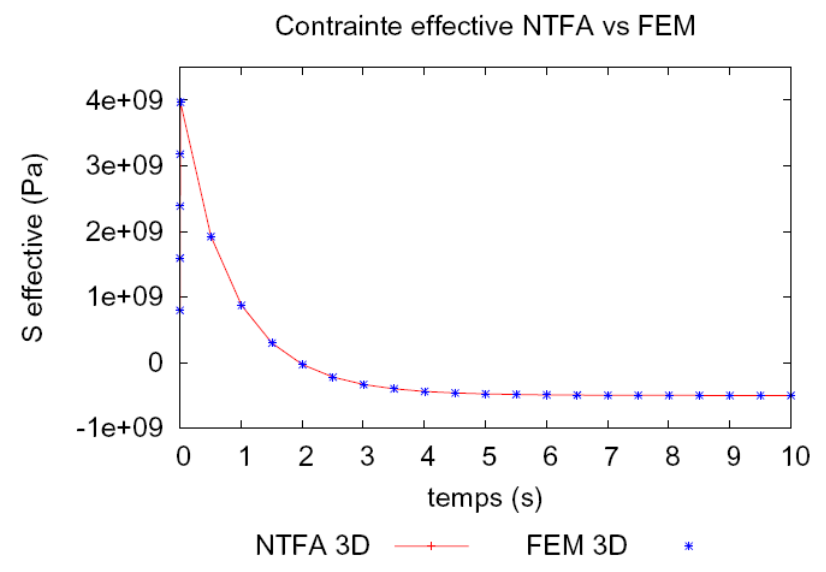

(b)

Fig. 4. Essais de fluage et relaxation sur la microstructure de la figure 2. (a) Essai de fluage sous contrainte uniaxiale $\Sigma_{33}=$ $100 \mathrm{MPa}$. (b) Essai de relaxation uniaxiale $E_{33}=2 \%$.

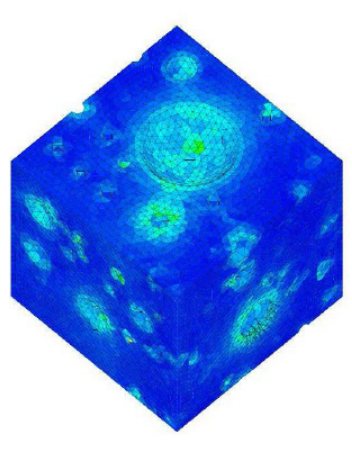

(a)

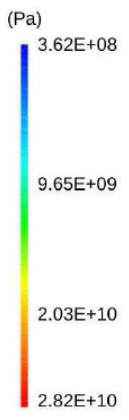

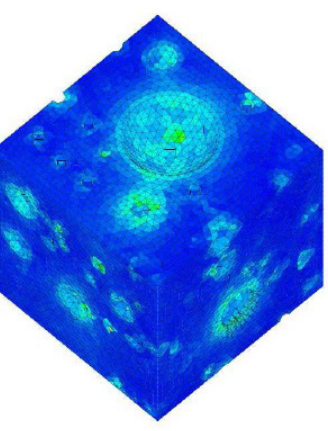

(b)

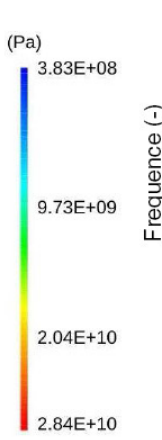

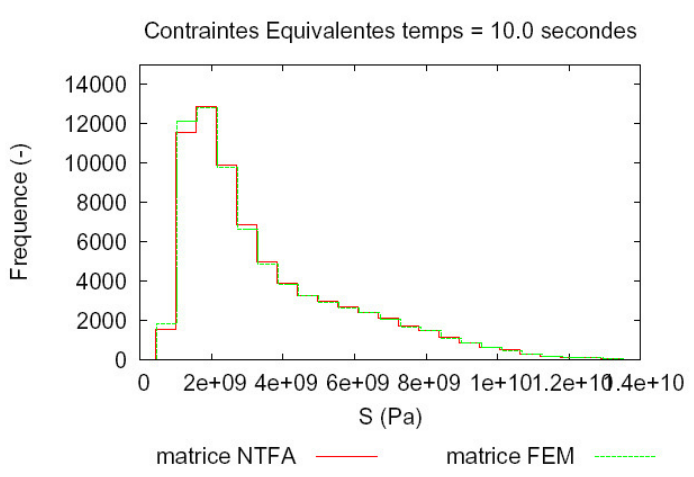

(c)

Fig. 5. Essai d'écrouissage numéro $8, t=10 \mathrm{~s}-\sigma_{\text {eq }}(\underline{x})$ NTFA vs. FEM - Phase matricielle. (a) NTFA isovaleurs. (b) FEM isovaleurs. (c) NTFA vs. FEM Distribution de $\sigma_{\text {eq }}(\underline{x})$ (von Mises).

Qualitativement, les isovaleurs de ce champ prédites par le modèle NTFA sont en bonne adéquation avec la prédiction des éléments-finis. La comparaison entre éléments-finis et NTFA de la distribution de ce champ de contrainte dans la phase matricielle (cf. Fig. 5c) confirme cette remarque. En effet, quantitativement la prédiction du modèle NTFA est excellente. L'erreur commise par le modèle NTFA sur la contrainte moyenne équivalente de la phase matricielle est égale à $+0,28 \%$, tandis que l'écart sur l'écart type est inférieur à $-0,81 \%$. Pour la phase inclusionnaire, des conclusions identiques ont pu être établies. Pour les essais de type fluage et relaxation, nous avons également observé une très bonne prédiction locale des différents champs de déformations ou de contraintes.

\section{Conclusions et perspectives}

Dans ce document, nous avons présenté les travaux théoriques et numériques qui ont été menés sur l'approche NTFA pour l'adapter au comportement du combustible MOX, à savoir un comportement élastoviscoplastique linéaire incompressible (l'incompressibilité ne touche que la partie viscoplastique) avec déformation libre. Comme [4-6], l'extension des approches par champs de transformation au $3 \mathrm{D}$ a été validée avec succès. La prise en compte de déformation libre, axe d'étude qui n'avait jamais été abordé jusqu'à présent côté NTFA, a également été validée avec succès aussi bien macroscopiquement que localement pour le comportement du combustible MOX et pour différents types de chargements (écrouissage, relaxation et fluage). En perspective de cette étude, nous envisageons les développements suivants sur le modèle NTFA : étude de l'aspect vieillissant et poro élasto-viscoplastique compressible non-linéaire sur un composite MOX 3D triphasé.

Remerciements. Ce travail a été réalisé dans le cadre de la convention de collaboration de recherche CEA-EDFCNRS 038279. 


\section{Références}

[1] J.-C. Michel, P. Suquet, Nonuniform transformation field analysis, Int. J. Solids Struct. 40 (2003) 6937-6955

[2] R. Largenton, V. Blanc, P. Thevenin, D. Baron, Simulation and modelling the heterogeneous effects of microstructure MOX fuels on their effective properties in nominal pressure water reactor conditions, Adv. Sci. Technol. 73 (2010) 9196

[3] S. Roussette, J.-C. Michel, P. Suquet, Nonuniform transformation field analysis of elastic-viscoplastic composites, Comp. Sci. Techn. 69 (2009) 22-27
[4] F. Fritzen, T. Bohlke, Three-dimensional finite element implementation of the nonuniform transformation field analysis, Int. J. Num. Methods Eng. 84 (2010) 803-829

[5] F. Fritzen, T. Bohlke, Nonuniform transformation field analysis of materials with morphological anisotropy, Compos. Sci. Technol., doi: 10.1016/j.compscitech.2010.12.013, 2011

[6] C. Oskay, J. Fish, Eigendeformation-based reduced order homogenization for failure analysis of heterogeneous materials, Comput. Methods Appl. Mech. Eng. 196 (2007) 1216-1243 